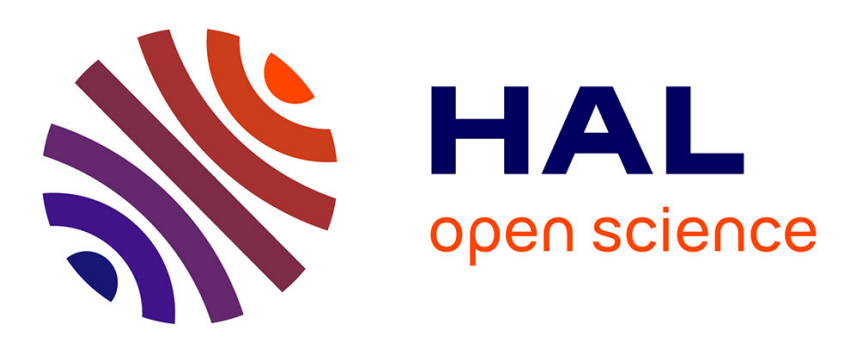

\title{
Appareils de mesures automatiques de frottement interne à moyenne et haute fréquences et à amplitude constante
}

\author{
G. Dedianne, D. Lemercier, P. Lemercier
}

\section{- To cite this version:}

G. Dedianne, D. Lemercier, P. Lemercier. Appareils de mesures automatiques de frottement interne à moyenne et haute fréquences et à amplitude constante. Revue de Physique Appliquée, 1969, 4 (3), pp.423-429. 10.1051/rphysap:0196900403042300 . jpa-00243307

\section{HAL Id: jpa-00243307 https://hal.science/jpa-00243307}

Submitted on 1 Jan 1969

HAL is a multi-disciplinary open access archive for the deposit and dissemination of scientific research documents, whether they are published or not. The documents may come from teaching and research institutions in France or abroad, or from public or private research centers.
L'archive ouverte pluridisciplinaire HAL, est destinée au dépôt et à la diffusion de documents scientifiques de niveau recherche, publiés ou non, émanant des établissements d'enseignement et de recherche français ou étrangers, des laboratoires publics ou privés. 


\title{
APPAREILS DE MESURES AUTOMATIQUES DE FROTTEMENT INTERNE A MOYENNE ET HAUTE FRÉQUENGES ET A AMPLITUDE CONSTANTE
}

\author{
Par G. DEDIANNE, Mme D. LEMERGIER et P. LEMERGIER, \\ Centre d'Études Nucléaires de Grenoble, Service de Physique du Solide et de Résonance Magnétique \\ et Laboratoire d'Électronique et de Technique de l'Informatique.
}

(Reçu le 24 janvier 1969, vévisé le 28 avril 1969.)

\begin{abstract}
Résumé. - Nous décrivons trois appareils de mesures de frottement interne à amplitude constante. Deux d'entre eux sont des pendules à lames fonctionnant l'un en torsion, l'autre en flexion dans la gamme de fréquence $20 \mathrm{~Hz}-1000 \mathrm{~Hz}$. Le troisième utilise des barreaux excités longitudinalement dans la gamme de fréquence $10 \mathrm{kHz}-50 \mathrm{kHz}$. Tous trois fonctionnent actuellement de $77^{\circ} \mathrm{K}$ à 500 oK. Ils sont entièrement automatiques et permettent des enregistrements continus du frottement interne et de la fréquence.
\end{abstract}

Abstract. - Three devices for constant amplitude measurement of internal friction are described. Two of these are blade pendulums operating in the frequency range 20-1 $000 \mathrm{cycles} / \mathrm{s}$, one of them in the torsional mode and the other in flexion. In the third apparatus a rod specimen is excited to longitudinal vibrations, in the 10-50 kilocycles frequency range. At present all three installations are working between $77 \mathrm{oK}$ and $500 \mathrm{oK}$. They are entirely automatic and permit continuous recording of frequency and internal friction.

Introduction. - Les trois appareils décrits ont été étudiés en vue de couvrir la gamme de fréquence $20 \mathrm{~Hz}-50 \mathrm{kHz}$ afin de relayer les pendules inversés automatiques [1], [2], utilisés à basse fréquence.

Une boucle électronique d'entretien permet de travailler à amplitude constante et de faire des enregistrements continus du frottement interne pendant les variations déterminées (montée linéaire, isotherme, isochrone) de température. Ce dispositif automatique permet de faire des mesures très longues (isothermes de 24 heures et plus) sans expérimentateur et de travailler la nuit (gain de temps et diminution des bruits d'origine mécanique). En outre, il est possible de suivre des variations rapides de frottement interne et de rendre visibles des pics fugitifs disparaissant rapidement dans le domaine de température de mesures.

Nous décrirons successivement les trois parties essentielles de chaque appareil : la partie mécanique, l'ensemble capteur-excitateur et l'électronique d'entretien. Les deux premières parties permettent des mesures classiques par décroissance ou largeur de bande. L'adjonction de l'électronique d'entretien permet des mesures continues et automatiques. Nous décrirons successivement deux dispositifs électroniques d'entretien, l'un fonctionnant dans la première gamme de fréquence, $20 \mathrm{~Hz}-1000 \mathrm{~Hz}$, et utilisé aussi bien pour les mesures en torsion que pour celles en flexion, l'autre fonctionnant dans la deuxième gamme de fréquence $10 \mathrm{kHz}-50 \mathrm{kHz}$ et utilisé pour les mesures sur des barreaux excités longitudinalement. Ges deux dispositifs diffèrent dans leur réalisation et seront décrits en détail dans le paragraphe "description des circuits », mais leur principe est analogue.

On peut montrer que la partie mécanique de la plupart des appareils de frottement interne peut être représentée avec une bonne approximation par une équation différentielle du second ordre du type :

$$
m \frac{\mathrm{d}^{2} x}{\mathrm{~d} t^{2}}+f \frac{\mathrm{d} x}{\mathrm{~d} t}+k x=F_{\mathrm{a}}
$$

$x$ étant le déplacement de l'échantillon, $f$ le terme de frottement interne (les frottements parasites devant être négligeables devant lui) et $F_{\mathrm{a}}$ la force appliquée. La mesure du frottement interne se fait par l'intermédiaire de celle de $Q^{-1}=\frac{f}{m \omega}$. Trois méthodes sont possibles suivant la valeur de $F_{\mathrm{a}}$ :

1) La force appliquée est nulle $\left(F_{\mathrm{a}}=0\right)$. La solution de (1) est :

$$
x=\chi_{m} \mathrm{e}^{-\alpha t} \cos \omega t
$$

avec $\alpha=\frac{f}{2 m}$ et $\omega=\sqrt{\frac{k}{m}\left(1-\frac{f^{2}}{4 k}\right)}$.

La mesure de $Q^{-1}$ est faite par décroissance libre. 
2) La force appliquée est sinusoïdale; le système vibre en oscillations forcées :

$$
F_{\mathrm{a}}=F_{0} \cos \omega t \text {. }
$$

La solution de (1) est :

$$
x=\chi_{m} \cos (\omega t+\Phi) .
$$

La mesure de $Q^{-1}$ se fait par la méthode dite de largeur de bande.

3) La force appliquée est fournie par une boucle électronique de gain $G$, contenant un déphaseur de $\frac{\pi}{2}$ :

$$
F_{\mathrm{a}}=G \frac{\mathrm{d} x}{\mathrm{~d} t} \text {. }
$$

L'équation (1) devient :

$$
m \frac{\mathrm{d}^{2} x}{\mathrm{~d} t^{2}}+(f-G) \frac{\mathrm{d} x}{\mathrm{~d} t}+k x=0 .
$$

Selon que $G$ est supérieur, égal ou inférieur à $f$, les oscillations sont croissantes, stationnaires ou décroissantes. La solution de (1) devient :

$$
x=\chi_{m} \mathrm{e}^{-\alpha t} \cos \omega t
$$

avec :

$$
\alpha=\frac{f-G}{2 m}
$$

$\frac{\mathrm{d} x}{\mathrm{~d} t}=-\chi_{m} \mathrm{e}^{-\alpha t}(\omega \sin \omega t+\alpha \cos \omega t)$

$$
=-\chi_{m} \mathrm{e}^{-\alpha t} \sin \left(\omega t+\operatorname{arctg} \frac{\alpha}{\omega}\right) \text {. }
$$

Le déphasage $\psi$ entre la force appliquée $\left(F_{\mathrm{a}}=G \frac{\mathrm{d} x}{\mathrm{~d} t}\right)$ et la déformation $\left(x=\chi_{m} \sin \left(\omega t+\frac{\pi}{2}\right)\right)$ est :

$$
\psi=\frac{\pi}{2}+\operatorname{arctg} \frac{\alpha}{\omega} \simeq \frac{\pi}{2}+\frac{\alpha}{\omega}=\frac{\pi}{2}+\frac{f-G}{2 m \omega} .
$$

Si $f \equiv G, \psi \equiv \frac{\pi}{2}$ (oscillations entretenues).

Nous utilisons dans tous nos appareils cette troisième méthode de mesure, qui, seule, donne une valeur instantanée du frottement interne par la mesure de $G$.

L'électronique associée aux différents appareils est schématisée figure 1. On remarquera les deux boucles : la première (échantillon-capteur-déphaseur-gain va-

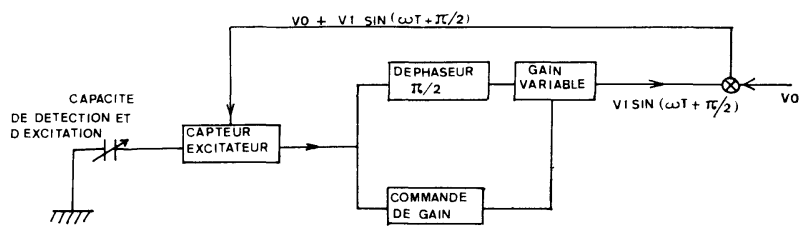

FIG. 1. - Schéma synoptique de principe des boucles électroniques d'entretien. riable-excitateur-échantillon) entretient les vibrations de l'échantillon en fournissant de l'énergie; la seconde boucle (commande de gain) maintient l'amplitude à un niveau déterminé $(G=f)$. Précisons le fonctionnement de la chaîne d'entretien. L'éprouvette est soumise à des vibrations entretenues à sa fréquence propre. Un capteur, de type capacitif, fournit une tension $V_{\mathrm{d}}=k_{1} \chi_{m}$ proportionnelle à l'amplitude des oscillations et de fréquence $\nu$. On applique à l'échantillon une tension $v$, somme d'une tension continue $V_{0}$ et d'une tension $V_{1}$, alternative, de fréquence $\nu$, obtenue à partir de la tension $V_{\mathrm{d}}$ déphasée de $\frac{\pi}{2}$ (la force et la déformation étant en quadrature) et convenablement amplifiée $\left(V_{1}=G V_{\mathrm{d}}\right)$ :

$$
v=V_{0}+V_{1} \sin 2 \pi v t \text {. }
$$

Dans ces conditions, la force appliquée est :

$$
F=\frac{\partial}{\partial e}\left(\frac{1}{2} c v^{2}\right)
$$

où $c$ est la capacité du condensateur éprouvetteexcitateur.

Il vient :

$$
\begin{aligned}
F=\frac{\partial c}{\partial e}\left(\frac { 1 } { 2 } \left(V_{0}^{2}\right.\right. & \left.+\frac{V_{1}^{2}}{2}\right) \\
& \left.+V_{0} V_{1} \sin 2 \pi \nu t-\frac{V_{1}^{2}}{4} \cos 4 \pi \nu t\right) .
\end{aligned}
$$

L'expression contient des termes aux fréquences $0 v$ et $2 \nu$. Si $V_{0}=0$, la force d'excitation est à la fréquence double; par contre, l'addition de $V_{0}$ fait apparaître un terme à la fréquence propre $\nu$ de l'échantillon et le choix du rapport $V_{0} / V_{1}$ permet de ne tenir compte que des deux premiers termes de la parenthèse de l'expression (2). Dans ces conditions, la force est de la forme :

$$
F=F_{0}+F_{1} \sin 2 \pi v t
$$

c'est-à-dire une force constante plus une force sinusoïdale d'amplitude proportionnelle à la tension alternative $v_{1}$ réinjectée.

Le travail fourni par cycle est :

$$
W=k_{2} V_{1} \chi_{m}
$$

L'énergie perdue par cycle est :

$$
\begin{gathered}
\Delta E=2 \pi E Q^{-1} \\
E=C \chi_{m}^{2}=\text { énergie élastique totale }
\end{gathered}
$$

d'où :

$$
\begin{gathered}
2 \pi E Q^{-1}=k_{2} V_{1} \chi_{m} \\
Q^{-1}=\frac{k_{2} G\left(k_{1} \chi_{m}\right) \chi_{m}}{2 \pi C \chi_{m}^{2}}=\frac{k_{1} k_{2}}{2 \pi C} G .
\end{gathered}
$$

La mesure de frottement interne se fera par mesure de gain $G$ ou encore par celle de la tension $V_{1}$ qui lui est proportionnelle quand $V_{\mathrm{d}}$ est maintenu constant (commande de gain). 
I. Appareil fonctionnant à moyenne fréquence. DESGRIPTION DU PENDULE DE FLEXION ( fig. 2). - L'appareil, dans son ensemble, présente des analogies avec ceux de Robinson [3], Benoit [4], Boch [5].

L'échantillon est une lame, de section rectangulaire,

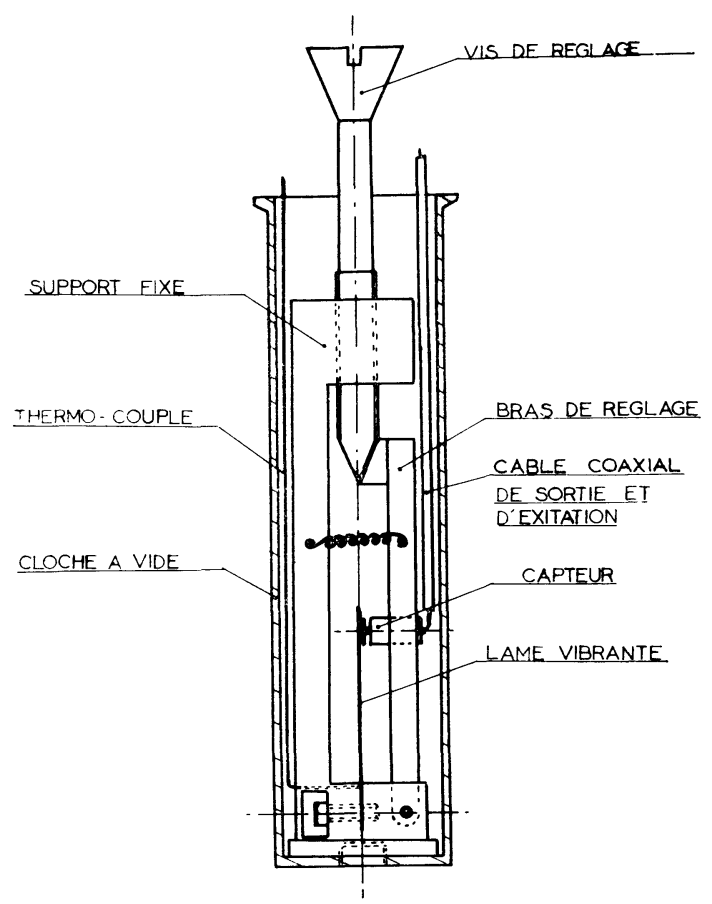

FIG. 2. - Pendule de flexion :

Schéma mécanique, échelle 0,4 .

encastrée à une extrémité et libre à l'autre. Sa fréquence propre de vibration, sur le mode fondamental, est :

$$
f=0,56 \frac{e}{L^{2}}\left(\frac{E}{12 \rho\left(1-\sigma^{2}\right)}\right)^{1 / 2}
$$

$e=$ épaisseur de la lame,

$L=$ longueur de la lame,

$\rho=$ densité de l'échantillon,

$\sigma=$ coefficient de Poisson,

$E=$ module de Young.

L'échantillon peut éventuellement vibrer à une fréquence inférieure si on le charge en bout.

L'appareil fonctionne sous vide secondaire pour éviter l'amortissement dû à l'air. On remarquera sur la figure 2 la présence d'un réglage extérieur de l'armature capteur-excitateur.

Le chauffage peut se faire soit par four classique, soit par bain thermostaté. Le montage est largement solidaire du fond de la cloche afin d'avoir une bonne conduction thermique. Les principaux avantages du chauffage par bain sont ici : la suppression totale du champ magnétique résiduel d'un four, la diminution de gradient de température, la rapidité du temps de montée $\left(7 \mathrm{mn}\right.$ pour $\left.250^{\circ}\right)$ qui permet le tracé d'isothermes et éventuellement d'isochrones (avec plusieurs bains).

Description Du PENDULE De TORSION ( $f$ ig. 3 et 4 ). L'échantillon est une lame encastrée à sa partie inférieure. Une palette, fixée perpendiculairement au plan de l'échantillon et à sa partie supérieure, constitue l'armature mobile du capteur. La palette ne peut pas vibrer en flexion car sa fréquence propre de vibration est très élevée (supérieure à $10 \mathrm{kHz}$ ), alors que la fréquence de l'oscillation est de l'ordre de $100 \mathrm{~Hz}$.

Nous avons constaté expérimentalement qu'il n'était

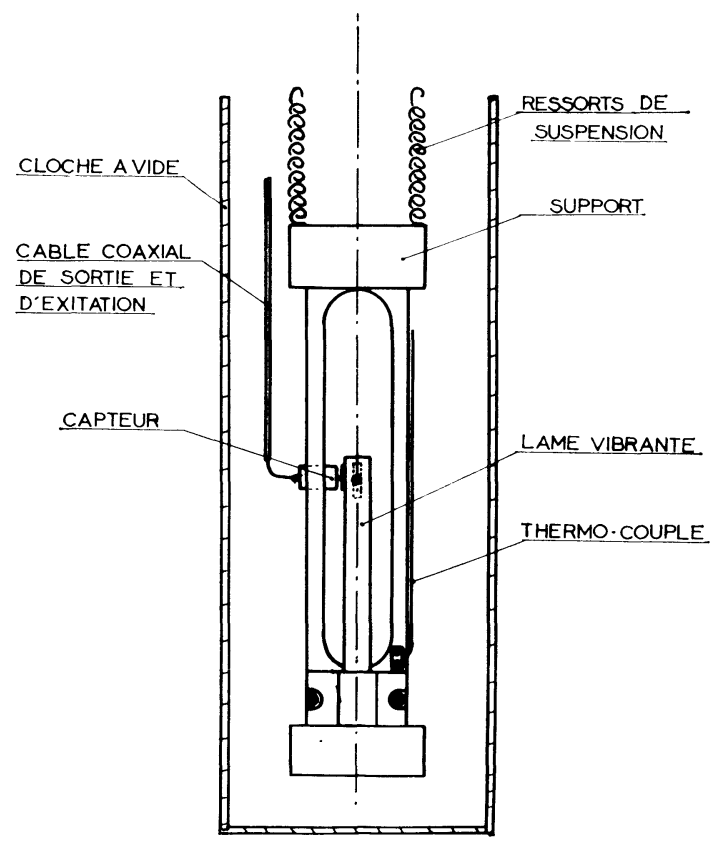

Fig. 3. - Pendule de torsion : Schéma mécanique, échelle 0,4 .

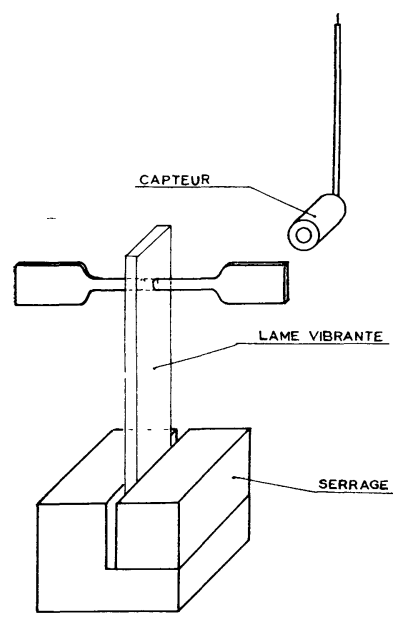

FIG. 4. - Pendule de torsion : Vue partielle schématique. 
pas nécessaire de travailler sous vide secondaire. Une faible pression de gaz (hélium par exemple) améliore considérablement les échanges thermiques et permet d'isoler le pendule de la cloche, c'est-à-dire des vibrations parasites extérieures transmises par la cloche.

Description du capteur excitateur ( fig. 5). Le capteur comprend un générateur VHF $(68 \mathrm{MHz})$ alimentant une ligne terminée par une capacité va-

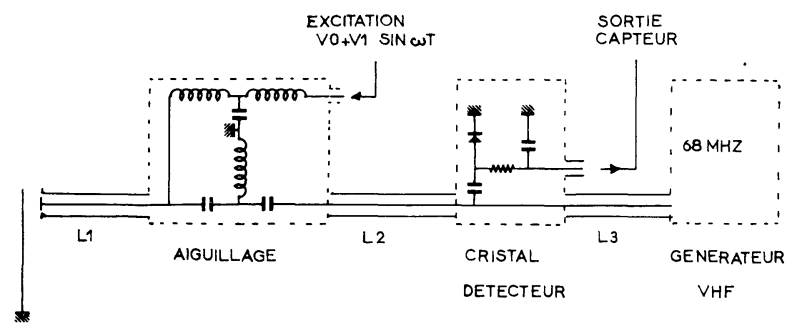

FIG. 5. - Schéma d'ensemble du capteur excitateur :

$\mathrm{L}_{1}, \mathrm{~L}_{2}, \mathrm{~L}_{3}=$ ligne coaxiale ; aiguillage $=$ filtres passe-haut et passe-bas séparant les deux fonctions $=$ capteur et excitateur.

riable : la capacité de mesure et un cristal détecteur. En effet, le conducteur central de la ligne est terminé par une petite pastille qui constitue l'armature fixe d'un condensateur variable. L'échantillon (lame vibrante) placé en regard constitue l'armature mobile. Le système d'ondes stationnaires établi dans la ligne se translate le long de celle-ci lorsque la capacité terminale varie, c'est-à-dire la distance entre l'objet dont on détecte le mouvement et le capteur. Le courant détecté par le cristal (fixé en un point de la ligne) fluctue suivant le déplacement des ondes stationnaires. En un point déterminé, donc, le courant détecté peut servir à représenter la position de l'objet. Il convient toutefois de bien positionner le cristal pour avoir une bonne linéarité et d'accorder la ligne pour avoir la sensibilité maximum.

La sensibilité est fonction de la distance et de la surface des armatures en regard. Dans l'application décrite ici, la tension détectée $V_{\mathrm{d}}$ est de $20 \mathrm{mV}$ crêtecrête par micron avec une distance entre armatures de $0,2 \mathrm{~mm}$. Le bruit est de l'ordre de quelques millivolts.

Les principaux avantages de ce capteur sont : sa grande sensibilité, sa très grande simplicité, la possibilité de le placer loin de l'électronique associée [6].

L'excitation est également de type capacitif et les deux fonctions capteur-excitateur sont réunies. La séparation s'effectue à l'aide d'un aiguillage constitué de filtres passe-bas et passe-haut ( fig. 5).

DESGRIPTION DES GIRGUITS POUR APPAREILS MOYENNE FRÉQUENCE. - Le schéma synoptique est présenté figure 6 .

Le signal détecté peut être maintenu constant à différents niveaux : 100, 200, 500 et $1000 \mathrm{mV}$ crête-

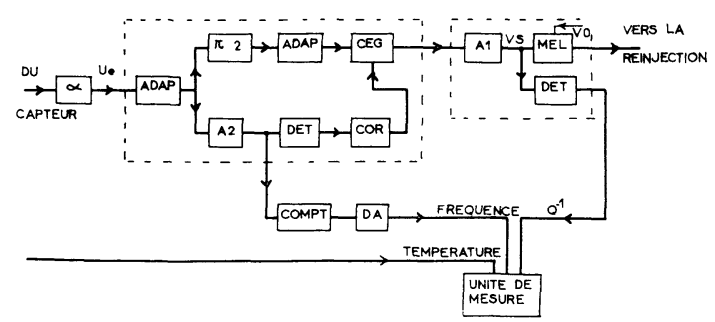

FIG. 6. - Schéma synoptique de l'électronique d'entretien et de mesure :

$\alpha=$ atténuateur variable ; adapteur $=$ étage adapteur d'impédance ; $\pi / 2=$ déphaseur de $\pi / 2 ; \mathrm{A}_{1}, \mathrm{~A}_{2}=$ amplificateurs ; $\mathrm{CEG}=$ contrôle électrique de gain ; $\mathrm{DE} T$ $=$ détection $; \mathrm{COR}=$ correcteur à retard de phase ; $\mathrm{MEL}=$ mélangeur $; \mathrm{COMPT}=$ compteur $; \mathrm{D} . \mathrm{A} .=$ convertisseur digital analogique.

crête. Un atténuateur variable $\alpha$ fournit toujours une tension d'entrée $U_{\mathrm{e}}=100 \mathrm{mV}$ crête-crête.

Nous avons montré que le déphasage entre la force et le déplacement était identiquement égal à $\frac{\pi}{2}$ pour un système bouclé. Le déphasage peut être obtenu grâce à un circuit dérivateur, un circuit intégrateur ou un circuit à module constant. Ce troisième circuit (voir fig. 7) a été choisi parce que son rapport signal sur bruit est indépendant de la fréquence et parce que la phase est réglable de façon continue grâce au potentiomètre situé dans l'émetteur du transistor de cet étage. On peut montrer que pour $R C \omega=1$ le déphasage est de $90^{\circ}$. Le réglage de la phase est utilisé au montage d'un nouvel échantillon (sa fréquence propre de vibration n'étant généralement pas la même que celle de l'échantillon précédent) et dans le cas d'un étalonnage par largeur de bande. Notons enfin qu'au cours d'un cycle thermique la fréquence propre de

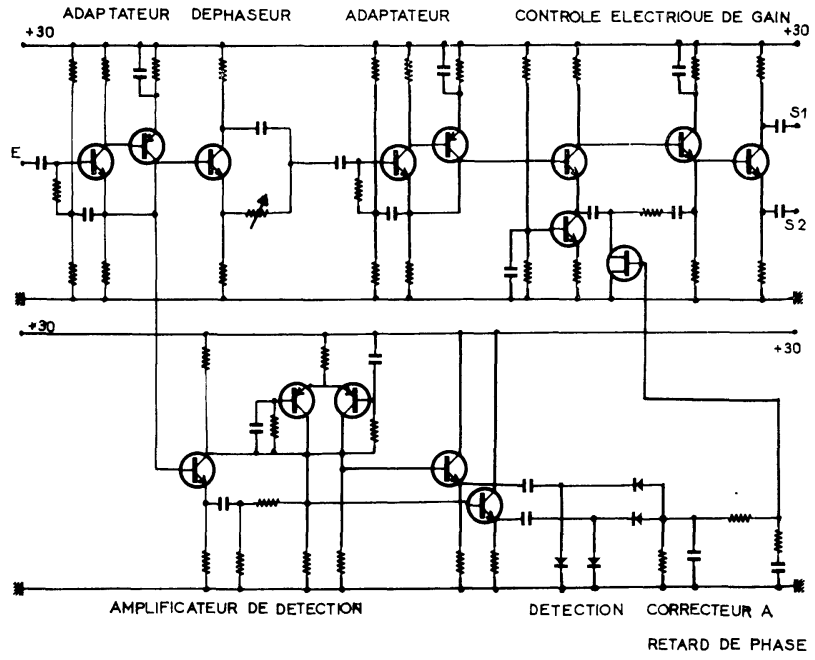

FIG. 7. - Schéma des principaux circuits de l'électronique d'entretien. 
vibration varie légèrement (variation des dimensions géométriques et éventuellement " effet de module »). Mais ces variations de fréquence sont très faibles (quelques pour cent au maximum) et, bien que le déphasage ait été réglé pour une fréquence déterminée $(R C \omega=1)$, le déphasage reste extrêmement voisin de $\frac{\pi}{2}$. Une seconde boucle $\left(A_{2}\right.$, détecteur et correcteur) contrôle le gain de la première boucle de façon à maintenir la tension d'entrée $U_{\mathrm{e}}$ constante.

La tension de sortie $V_{\mathrm{s}}$ est réinjectée après superposition avec une tension continue $V_{0}=250 \mathrm{~V}$ régulée.

On a vu, dans l'introduction, que la tension $V_{\mathrm{s}}$ est proportionnelle au frottement interne $Q^{-1}$. Après détection, elle peut être enregistrée de façon continue. L'étalonnage est fait par décroissance ou largeur de bande. Un compteur suivi d'un convertisseur digital analogique permet la mesure et l'enregistrement continu de la fréquence. Les principaux circuits sont détaillés figure 7 .

II. Appareil fonctionnant de 10 à 50 kHz. - PARTIE MÉGANIQUE. - Comme l'appareil de Cabarat [7], nous utilisons la résonance propre d'un barreau fixé en son milieu et vibrant à demi-longueur d'onde. Sa fréquence est imposée par sa longueur et par la vitesse de propagation des ondes dans le matériau utilisé. L'échantillon est placé sous vide pour minimiser l'amortissement dû à la lame d'air comprise entre le capteur et l'extrémité du barreau. Le chauffage est obtenu par un four bobine double. Le capteur et l'excitation étant capacitifs, la dilatation du barreau fait varier leurs sensibilités. Nous avons regroupé ces deux fonctions en un seul élément comme l'avaient fait Bordoni [8], Southgate [9] et Hinton [10]. Nous verrons dans la description de la partie électronique qu'il est très facile de réguler la position de cet élément à l'aide d'un moteur placé à l'extérieur de l'enceinte à vide.

Cet ensemble mécanique permet le montage de l'échantillon à froid, il comprend deux parties principales (voir fig. 8) :

- La platine supérieure sur laquelle sont fixées les sorties des fours, du capteur, le thermocouple de mesure de la température de l'échantillon. De plus, le moteur de régulation, le capteur, le support de celui-ci et les trois tubes de guidage qui peuvent être chauffés sont solidaires de la platine (tous ces éléments sont en traits forts sur la figure 8).

- La partie inférieure qui comprend : le support de l'échantillon, le four et les trois tiges de positionnement (en traits fins sur la figure 8).

L'échantillon est monté sur la partie inférieure qui est elle-même dans la cloche pleine d'azote. Le tout est fixé sous la platine, les trois tiges positionnant l'échantillon par rapport au capteur. Nous pompons alors l'azote, tout en chauffant le haut des tiges de

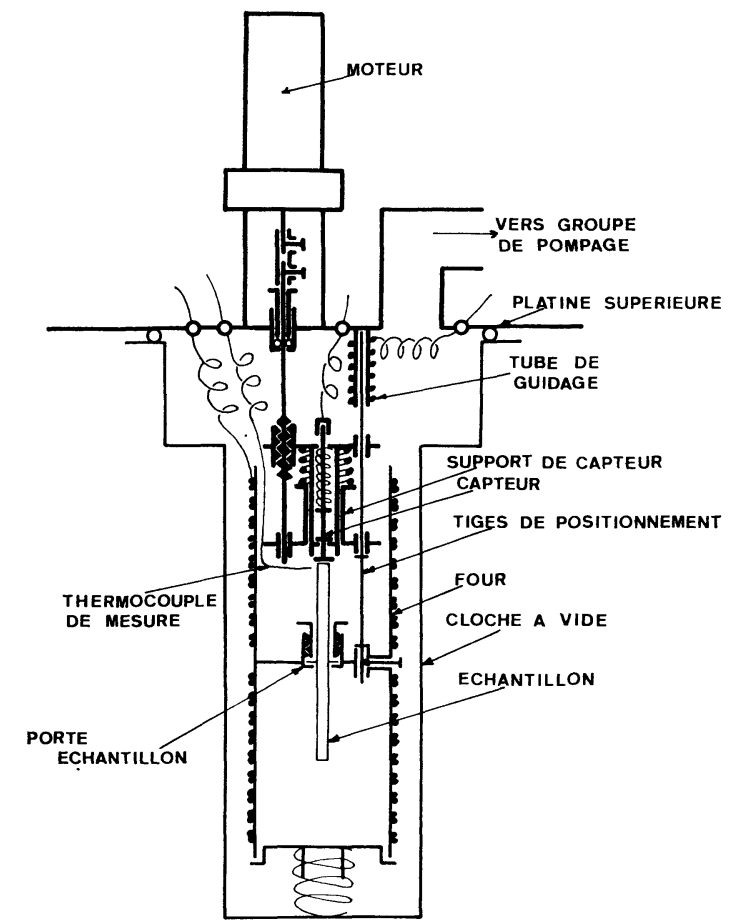

Fig. 8. - Schéma mécanique de l'appareil de mesure de frottement interne à haute fréquence, échelle 0,2 .

guidage qui a givré pendant le montage de l'échantillon.

Le barreau peut être fixé par trois vis pointeaux, par deux demi-bagues ou par un disque usiné dans la masse de l'échantillon. Avec cette dernière fixation, nous avons obtenu un coefficient de surtension de $10^{6}$ pour un échantillon d'AG1. Pour une variation de pression de $1 \mathrm{~mm}$ de $\mathrm{Hg}$ à $10^{-5} \mathrm{~mm}$ de $\mathrm{Hg}$, ce coefficient de surtension ne s'améliore que de $5 \%$ au maximum. Ceci nous permet de travailler avec une faible pression de gaz d'échange. Nous obtenons un gradient de température dans l'échantillon inférieur à $1^{\circ} \mathrm{C}$ et une différence entre l'échantillon et le thermocouple de mesure de $1^{\circ} \mathrm{C}$.

Capteur-Réinjegtion. - Le capteur est de type capacitif et fonctionne en modulation d'amplitude (voir fig. 9).

Un générateur $\mathrm{VHF}$ de $142 \mathrm{MHz}$ excite un premier circuit oscillant à travers un filtre en $\pi$, la capacité du capteur est ramenée en parallèle sur un second circuit oscillant couplé au premier par une ligne coaxiale de longueur $n \lambda / 2$. Nous réglons ces deux circuits de telle sorte que la fréquence de $142 \mathrm{MHz}$ soit au point d'inflexion de leur courbe de résonance. Le générateur VHF piloté par un quartz est alimenté par une tension stabilisée. Le filtre en $\pi$ adapte l'ensemble sur le générateur VHF.

Si l'on fait varier sinusoïdalement la capacité du capteur, la courbe de résonance des circuits oscillants 


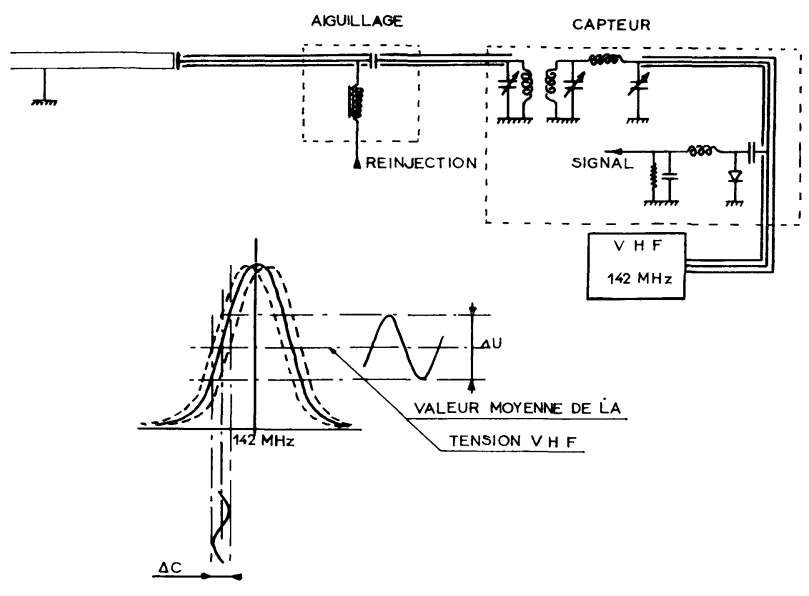

FIG. 9. - Schéma d'ensemble et principe de fonctionnement du capteur et de l'aiguillage.

se déplace de la même façon, et l'on produit une modulation de la tension VHF. Nous détectons cette modulation, et aussi la valeur moyenne de la tension VHF. Celle-ci traduit la position du capteur par rapport à l'échantillon, et nous permet de réguler cette distance.

Le capteur a une sensibilité de $40 \mathrm{mV}$ par micron à une distance moyenne de $0,25 \mathrm{~mm}$, avec un bruit inférieur à $1 \mathrm{mV}$. Il peut être placé très loin de la partie électronique, sans grand affaiblissement de sa sensibilité.

L'excitation est du type électrostatique pour travailler sur tous les matériaux conducteurs. La tension de réinjection de la forme $V=V_{0}+V_{1} \cos \omega t$ est appliquée sur l'électrode qui sert aussi au capteur. Un aiguillage formé d'une self de choc et d'une capacité évite que la tension VHF aille vers l'étage final d'amplification et empêche un court-circuit de la tension de réinjection par le second circuit oscillant.

Élegtronique D'ENTRETIEN ET RÉGULATION DE POSITION DU GAPTEUR. - Voir schéma synoptique, figure 10.

L'électronique d'entretien comprend deux boucles :

- La première maintient le barreau en oscillations; elle est constituée par :

- l'amplificateur $\mathrm{A}_{1}$,

- la commande électrique CEG de gain, ayant 1000 de dynamique,

- le déphaseur de $\pi / 2$ qui compose le déphasage entre la tension de réinjection et le signal détecté,

- l'amplificateur $\mathrm{A}_{2}$,

- le mélangeur $M$ qui superpose à la tension continue stabilisée $V_{0}=300 \mathrm{~V}$ la tension de sortie de $A_{2}$.

Tous ces éléments ont une bande passante de $100 \mathrm{kHz}$.

- La seconde maintient constante l'amplitude de vibration des barreaux; elle comprend :

- l'amplificateur $A_{3}$, dont le gain peut varier et fait ainsi varier l'amplitude de vibration,

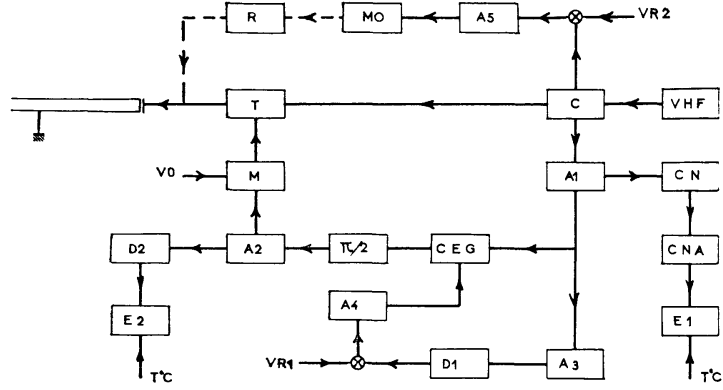

FIG. 10. - Schéma synoptique complet de l'électronique d'entretien et de mesure à haute fréquence :

$\mathrm{VHF}=$ générateur $142 \mathrm{MHz} ; \mathrm{C}=$ capteur $; \mathrm{T}=$ aiguil lage $; M=$ mélangeur ; $A_{1}, A_{2}, A_{3}, A_{4}, A_{5}=$ amplificateurs ; $\mathrm{CEG}=$ commande électrique de gain ; $\pi / 2=$ déphaseur $\pi / 2 ; D_{1}, D_{2}=$ détections $; C N=$ compteur numérique ; $\mathrm{CNA}=$ convertisseur numérique analogique $; \mathrm{E}_{1}, \mathrm{E}_{2}=$ enregistreurs $; \mathrm{MO}=$ moteur Birotax $\mathrm{R}=$ réducteur $1 / 3000$.

- la détection $\mathrm{D}_{1}$,

- l'amplificateur différentiel $\mathrm{A}_{4}$ bouclé en intégrateur à intégration limitée,

- la commande électrique de gain.

Les principaux éléments de ces boucles sont détaillés figure 11.

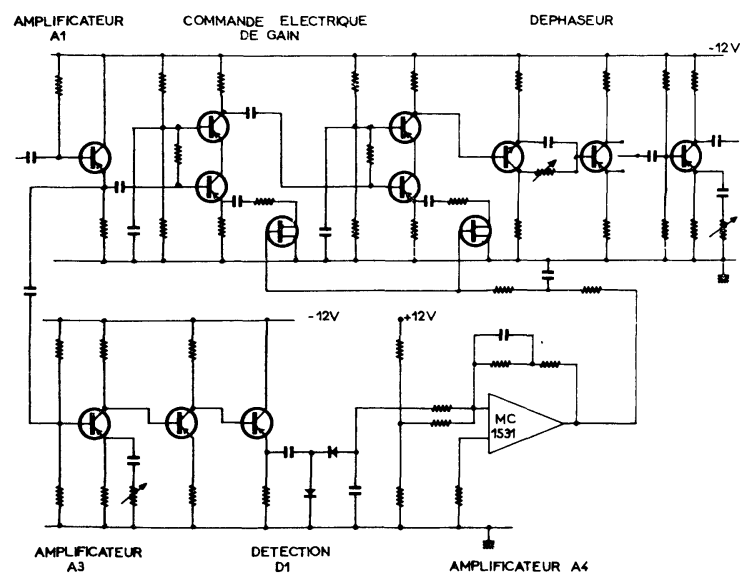

FIG. 11. - Schéma des principaux circuits de l'électronique d'entretien.

La régulation de position du capteur se fait par la boucle suivante :

- l'amplificateur différentiel $A_{5}$ bouclé par un filtre passe-haut pour améliorer la stabilité,

- le moteur MO Birotax type V,

- le réducteur $\mathrm{R} \mathrm{1/3000.}$

La mesure de $Q^{-1}$ se fait par la mesure de la tension de sortie de $\mathrm{A}_{2}$ qui est détectée par $\mathrm{D}_{2}$, après un étalonnage soit par largeur de bande, soit par décroissance. La précision de la mesure de $Q^{-1}$ est liée à la précision 
de la régulation de position du capteur égale à $\pm 0,25 \mu$. La distance capteur-échantillon étant en moyenne de $0,2 \mathrm{~mm}$, l'erreur résultante sera très faible quelle que soit la valeur de $Q^{-1}$.

III. Mesures. - Les appareils décrits sont utilisés pour plusieurs études. A titre d'exemple, nous donnons, figures 12 et 13, deux enregistrements. Le premier (pic de carbone dans le fer après remise en solution et

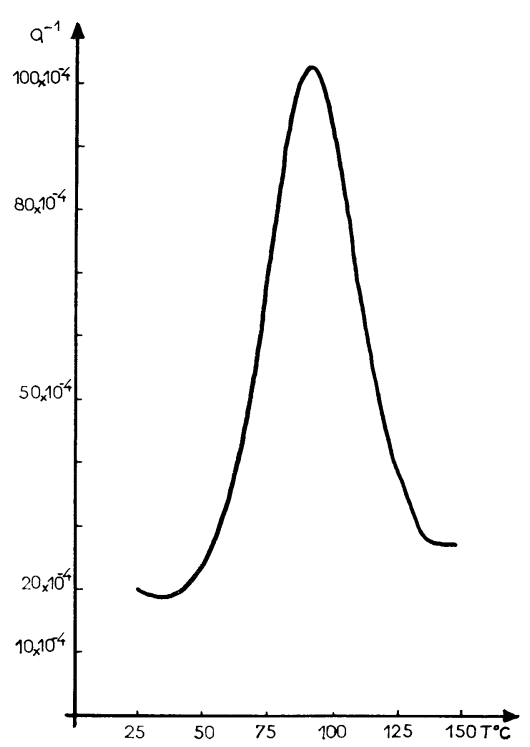

FIG. 12. - Pic du carbone dans le fer $\alpha$ fusion de zone recuit à $750^{\circ} \mathrm{C}$ et trempé dans l'eau glacée : enregistrement effectué à l'aide du pendule de flexion à $110 \mathrm{~Hz}$.

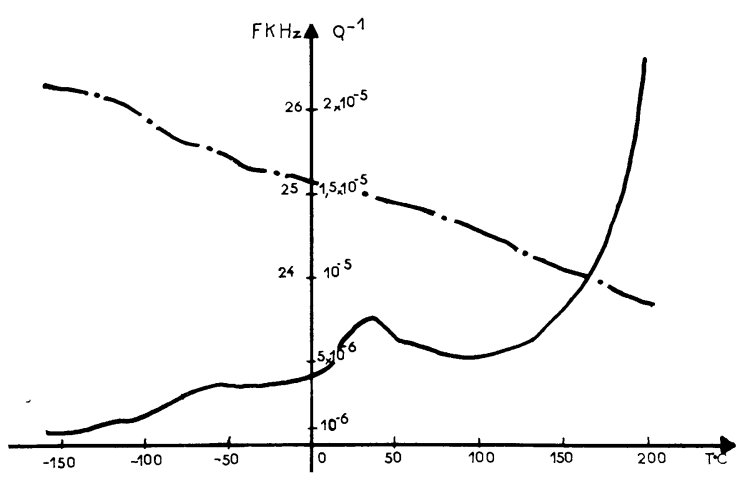

FIG. 13. - Frottement interne et fréquence d'un barreau d'AlMg $(1 \%)$ très pur recuit et trempé de $520^{\circ} \mathrm{C}$ dans l'azote liquide (montage à froid).

trempe) a été réalisé à l'aide de l'appareil de flexion à $110 \mathrm{~Hz}$; le second (frottement interne et fréquence d'un échantillon d'aluminium-magnésium $1 \%$ trempé) a été effectué à haute fréquence. Le fond continu très bas de cet appareil devra permettre l'étude de phénomènes très fins.

Les appareils fonctionnant à moyenne fréquence font partie de la thèse de Mme D. Lemercier (DocteurIngénieur, Grenoble, 1969); l'appareil fonctionnant en haute fréquence fait l'objet de la thèse de M. G. Dedianne (Docteur-Ingénieur, Grenoble, 1969).

Remerciements. - Nous remercions M. A. Salvi pour ses précieux conseils et MM. M. Bof, G. Casali et $\mathrm{A}$. Chapuis qui ont contribué à la réalisation des appareils.

\section{BIBLIOGRAPHIE}

[1] Salvi (A.), Dautreppe (D.) et Friess (E.), The Review of Scientific Instruments, 1965, 36, 198.

[2] Salvi (A.), Dautreppe (D.) et Friess (E.), Brevet no PV 936-466, 1963, U.S. Patent.

[3] Robinson (D. W.), Journal of Scientific Instruments, 1965, 32, 2.

[4] Benoit (W.), Thèse, Université de Lausanne, 1968.

[5] BLoch (Ph.) et DE Fougueit (J.), C. R. Acad. Sci. Paris, 1966, 260, 3957.
[6] LEMERCIER (D.) et LEMERCIER (P.), Brevet no PV 72735, 1966, U.S. Patent.

[7] Cabarat (R.), Revue de Métallurgie, 1949, XLVI, 617.

[8] Bordoni (P. G.), The Journal of Acoustical Society of America, 1954, 36, 495.

[9] SouthgaTE (P. D.), Journal of Scientific Instruments, 1959, 36, 284.

[10] Hinton (T.), The Review of Scientific Instruments, $1965,36,114$ 